International Journal of Engineering \& Technology, $7(4.11)(2018) 44-48$
International Journal of Engineering \& Technology
SPC
Website: www.sciencepubco.com/index.php/IJET
Research paper

\title{
EEG Calmness Index Establishment Using Computational of Z-Score
}

\author{
S. A. M. Aris ${ }^{1}$, N. A. Bani ${ }^{1}$, M. N. Muhtazaruddin ${ }^{1}$, M. N. Taib ${ }^{2}$ \\ ${ }^{1}$ Razak Faculty of Technology and Informatics, Universiti Teknologi Malaysia, Kuala Lumpur, Malaysia \\ ${ }^{2}$ Faculty of Electrical Engineering, Universiti Teknologi MARA, Shah Alam, Selangor, Malaysia \\ *Corresponding author E-mail: armiza.kl@utm.my
}

\begin{abstract}
A lot of useful information can be obtained through observation of the electroencephalogram (EEG) signal such as the human psychophysiology. It has been proven that EEG is handy in human diagnosis and tools to observe the brain condition. The study aims to establish a calmness index, which can differentiate the calmness level of an individual. Alpha waves were selected as the data features and computed into asymmetry index. The data features were clustered using Fuzzy C-Means (FCM) and resulted in three clusters. Wilcoxon Signed Ranks test was applied to determine the significance of the data features clustered by FCM. The Z-score obtained successfully distinguish three level of calmness index from the lower index until the higher index. With the advancement of signal processing techniques, the feature extractions for calmness index establishment computation is achievable.
\end{abstract}

Keywords: EEG; calmness index; z-score; alpha waves; Wilcoxon signed ranks test.

\section{Introduction}

\subsection{Brainwaves}

Brain waves are the patterns of neuronal electrical activities recorded by EEG. It is generated by synaptic activities of the brain neurons and can be captured at the surface of the cortex. Generally, brain waves are sinusoidal form and can be categorized into four types of frequencies known as the delta, theta, alpha, and beta. The four sub-bands are usually emphasized in cognitive research study either involves the normal or abnormal conditions. There is also another EEG band known as gamma band, which is also part of EEG signal with a frequency range from $30 \mathrm{~Hz}$ to $45 \mathrm{~Hz}$. Gamma waves are generally used for verification of certain brain diseases.

Delta wave usually presented at $4 \mathrm{~Hz}$ or below are frequently perceived during deep sleep of healthy adults and found in children below the age of one year [1]. Delta waves were also visible in the dementia patients [2] and were apparent in stroke patients [3]. Theta wave which lies between $4 \mathrm{~Hz}$ and $8 \mathrm{~Hz}$ are observed during drowsiness and sleep in adults and is also apparent in infants and children. However, the emergence of higher theta wave during wakefulness in adults implies irregular conditions and sometimes known as an indicator of mental attention [4]. Alpha wave is usually noticed in adults during relaxed wakefulness with the frequency range of $8 \mathrm{~Hz}$ to $13 \mathrm{~Hz}$. Generally, it can be generated by merely closing the eyes. Lastly, beta wave having a frequency range of $13 \mathrm{~Hz}$ to $30 \mathrm{~Hz}$ is generated in adults who are in the state of alertness or anxiety [5].

Alpha waves of EEG signal are usually chosen as the presence of alpha waves is obvious when the eyes are closed, and it disappears when the eyes are opened or when paying attention to any related brain activities. Consequently, alpha waves have been a sign of a relaxed awareness exclusive of any attentiveness. This situation was initially discovered by [6] that a sudden suspension of alpha waveforms occurred when an individual opens his eyes. Relaxed wakefulness with eyes-closed has also been treated as one of the fundamentals of electro-physiological phenomena, which has become a sign of calmness [7].

\subsection{Cognitive Ability}

Interest in the brain function and its relation to the associated cognitive has led to a drastic change in the use of tools and features. Studies on the categorization of the brain regions are dependent on the needs and goals of the study. One of the earliest types of categorization is the activations of any sides of the brain hemispheres towards specific stimulus, which is also known as the brain asymmetry. The term asymmetry, also known as laterality refers to a mechanism which shows the reflections of the left-right differences in psychology and the neurosciences. This is done by comparing the activation levels from the right-frontal versus the left-frontal cortical areas which can be accessed from the EEG recordings. To determine which side of cortical is more dominant, difference scores are broadly used in the research. Many scholars used brain asymmetry to recognize emotional significance from selected features to determine the emotions.

A lot of useful information can be obtained from EEG signal such as during the observation of the sedative level of a patient [8], distinguish patients with stress [9], depression [10] and dementia [11]. One of the latest EEG research, Z-score was utilized in EEG biofeedback for detecting the brain dysfunction [12]. Z-score can give relative scores of individuals in a distribution. Z-scores are computed by taking the difference between score and the mean divided by the standard deviation, which will provide either positive or negative scores.

Computing Z-scores as with many statistical measures depends upon whether one is computing the test for a population or a sample. The Z-scores and standard curves are usually related. In some 
inferential statistics, Z-score plays a vital role in the statistical argumentation. The advantage of using $\mathrm{Z}$-score is that it standardized two different distributions, which can then be compared. To some extent, Z-scores has been used as one of the methods in indexing the subjects based on the Intelligent Quotient (IQ) level [13] via EEG spectral density. In another study, Z-scores has been employed in recognition of the significant chemical compounds of the agarwood oils [14] and the identification of the agarwood substance that contributes the oil's fragrance. Also, Z-scores normalization is one of the relevance method used in metasearch study. However, the scores in metasearch must be in Gaussian distribution for the results of the Z-scores to be applicable [15]. The different samples and conditions of gene expressions were compared via $\mathrm{Z}$-score and some other tests, where the Z-score obtained has shown convincing results in predicting changes in the genes as compared to other test methods [16]. Thus, it is undoubtedly true that Z-score can be used as identification tools in selecting or indexing the data.

\section{Methodology}

The methods and resources used in the study were carefully chosen to obtain the real characteristics of EEG signals. The flow chart in Fig. 1 provides the flow of experiments methodically prepared for the study. The work comprises of the collection of EEG data, signal pre-processing, extraction of EEG feature using alpha wave, clustering of the EEG features using Fuzzy C-Means (FCM), EEG pattern classification and lastly is the EEG calmness indexing.

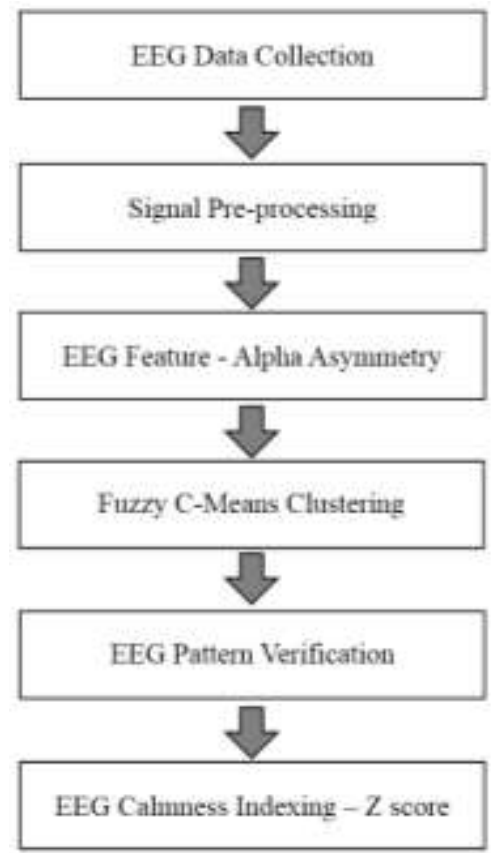

Fig. 1: Flow of research methods

\subsection{EEG Data Collection}

Forty-Three University students were involved in this study, their age ranging from 22 to 24 years old. Their involvement in the experiments is on their own motivation. All participants are right handed and were not under any medications. The subjects were explained regarding the experiments and were asked to desist from taking caffeine for 24 hours prior to the experiments. After the full explanation of the experimental procedures, they gave their written consent to participate in the experiments.

In this study, two pairing channels placing at Fp1-A1 and Fp2-A2 were chosen. As shown in Fig. 2, the paired electrodes at Fp1 and A1 are placed at the frontal of left hemisphere and left earlobe respectively and the paired electrodes at $\mathrm{Fp} 2$ and $\mathrm{A} 2$ are placed at the frontal of the right hemisphere and right earlobe respectively while the ground electrode is located at Fpz. The electrodes' impedance is maintained below $5 \mathrm{k} \Omega$ using $\mathrm{Z}$-checker equipment.

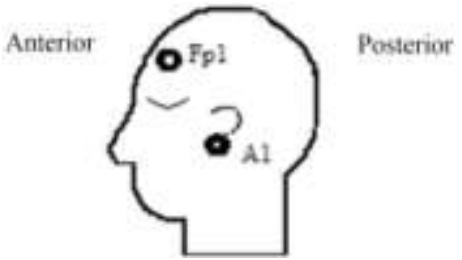

Fig. 2: Electrodes placement

The experiments protocol was set as in Table 1 . The relaxed state was set to 3.5 minutes of recording. Then, subjects were given a break for 15 minutes before starting the non-relaxed recording session.

Table 1: Experiments protocol

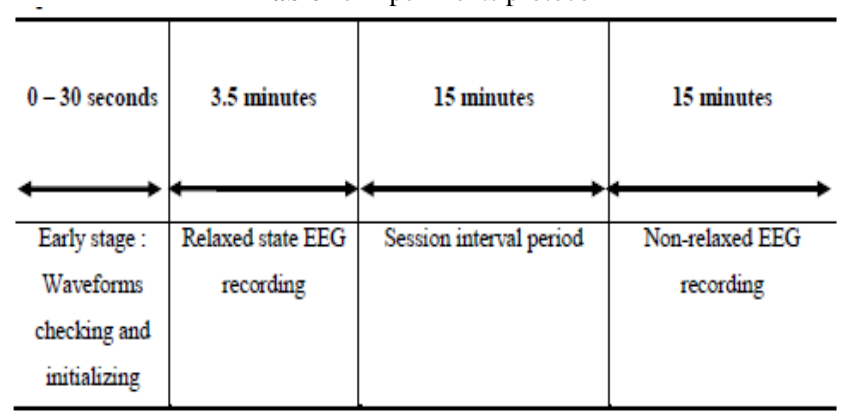

Two brain conditions were selected in the study named relaxed state and non-relaxed state. Relaxed state is synonymous with the resting state. During the data recording, the participants rest on a comfortable chair in a soundless room with dim lights and were asked to close their eyes during the EEG recording. Another type of brain stimulus used in the study was the mental task test. The participants were requested to answer to a non-verbal and logical thinking task test, which the idea was taken from the Raven Test. Questions are designed so that users can interact directly with the computer. The purpose of mental task test is to engage the subjects' brain into a working state, so that a contrary condition than the relaxed state is achieved.

\subsection{EEG Pre-Processing}

The EEG data that was recorded during relaxed and non-relaxed states were pre-processed. Only three minutes were taken from each of the EEG recorded signals for analysis. The 15 seconds segments at the beginning and ending of the filtered EEG signals were excluded in the analysis as it is always contaminated with noise. FFT was chosen to transform the EEG signals into spectral density and the EEG activities can also be quantified in a frequency band. FFT was selected as it gives faster computation time and involves less data storage [17]. Power spectral density of the signal can be obtained using the modulus-squared of the discrete Fourier transform with Hamming window and 50\% overlap. These settings were used as to follow the method used in Welch's. The right and left channels were converted to frequency domain before conducting further analysis.

The EEG signals must be filtered into sub-bands before it can be further analyzed and extracted. Frequency spectrums which are in power spectral density (PSD) were computed into ESD. The ESD values were calculated from the area under the PSD curve for alpha band $(8-12 \mathrm{~Hz})$. 


\subsection{EEG Feature - Alpha Asymmetry}

Asymmetry index (AsI) was taken at the alpha band and was computed in (1), which consist of relaxed state and non-relaxed state data features were prepared.

$$
A s I=\ln \left(E S D R_{\text {mean }}\right)-\ln \left(E S D L_{\text {mean }}\right)
$$

where $\ln \left(E S D R_{\text {mean }}\right)$ is the natural log of mean ESD at the right channel and $\ln \left(E S D L_{\text {mean }}\right)$ is the natural log of mean ESD at the left channel. This equation was derived from the difference of energy computed at the right lead with the energy computed at the left lead in the study.

\subsection{Fuzzy C-Means Clustering}

Fuzzy C-means (FCM) was used in the study to segregate the approximate EEG data into related groups. The AsI data during relaxed and non-relaxed states were set as the data points in FCM, subsequent after the cluster center searched through the subtractive clustering. Subtractive clustering (SC) is a type of clustering technique that is basically used to pre-determine the number of cluster that is set in FCM. AsI was selected as the data points to be generated by SC. The data points were normalized before it was transformed into .dat extension. The transformed data were used in the SC to generate the highest potential cluster center from each number found in the tabulated data. The highest potential cluster center is nominated as the first cluster center. Then, the process repeats to find for the next cluster center with the remaining data points until a sufficient number of clusters are found.

The FCM process starts with transforming the data into membership grades and initialized into appropriate matrix, U. Then, the initial clusters centers were computed and the iteration starts until the objective function reached its minimum to suits with the optimal position of the clusters. Every time this is done, the membership grades and the cluster centers will be updated. The iterations stop when the maximum number of iterations achieved or two consecutive objective functions have no more improvement noticed.

\subsection{EEG Pattern Verification}

The data features which was selected by FCM need to be verified. Thus, box plot was selected to compare the strength of the relaxed and non-relaxed states within a cluster. The box plot can show extreme values and the middle data value ranges. The minimum and the maximum values are the smallest and the largest values respectively within the data respectively. The box plot could show the difference of two groups clearly and it is also easy to interpret the pattern of the brain between relaxed and non-relaxed.

The Wilcoxon signed rank test is an alternative test to the parametric paired sample t-test. In the study, Wilcoxon signed rank test were chosen because the test provides a powerful test in perceiving a difference between either two small samples or large samples [18]. The Wilcoxon non-parametric test utilizes the W test statistic. The hypothesis testing via Wilcoxon signed rank test presented a quicker critical value of z-value. The z-value or the zratio must be 1.96 or higher to reject the null hypothesis. Thus, the relative efficiency of Wilcoxon test must achieve at least an asymptotic of $95.5 \%$, so that it is comparable with the t-test parametric test [19].

\subsection{EEG Calmness Indexing - Z Score}

Reviews have also supported that indexing does have benefits and has become important. There are many techniques applied to establish index and Z-score is one of them. The Z-score is very suitable to be used in comparing between different populations or samples, and this includes index establishment. In a normal data distribution, one of the major components that are also measured is the Z-score, which is used specially to support the statistical argumentation. The basic expression of a Z-score is

$$
z=\frac{x-\mu}{\sigma}
$$

Here, $x$ is the data, $\mu$ is the population mean and $\sigma$ is the standard deviation. If $x>\mu$, then the Z-score will be positive and if $x<\mu$, then the Z-score will be negative. Zero Z-score gives a meaning that both the data value and the mean have the same values.

Basically, Z-score measure the distance of an observation from above and below the mean. The general relationship between a normal distributions curve is illustrated in Fig. 3. Within the Zscore distribution, the mean is always zero with a minimal standard deviation is 1 . As showed in the Fig. 3, approximately $34 \%$ of the scores fall below and above the mean. This means $68 \%$ of the samples fall between $-1 \sigma$ and $+1 \sigma$. The score that falls between $2 \sigma$ and $+2 \sigma$ has $95.5 \%$ and the score that falls between $-3 \sigma$ and $+3 \sigma$ is $99.7 \%$. The Z-score characteristics can be summarized as that:

- The areas between $Z=-1 \sigma$ and $+1 \sigma$ is equal to $68.27 \%$. This means that $68.27 \%$ of the measurements will have a $\mathrm{Z}$ score between -1 and +1 .

- The areas between $Z=-2 \sigma$ and $+2 \sigma$ is equal to $95.45 \%$.

- The areas between $Z=-3 \sigma$ and $+3 \sigma$ is equal to $99.73 \%$.

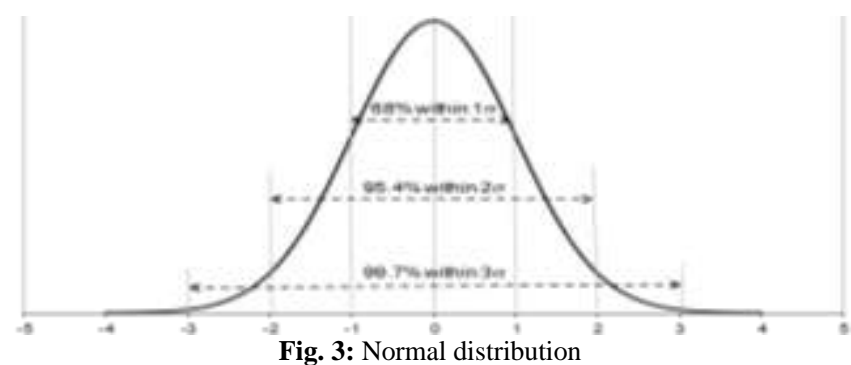

\section{Results and Discussion}

\subsection{EEG Features - Alpha Asymmetry}

Asymmetry index (AsI) was used as the EEG features to categorize calmness. Fig. 4 shows the computed AsI values, either positive or negative that were separated by the relaxed state and the non-relaxed state. The relaxed state and the non-relaxed state were plotted based from the positive and negative values achieved, which referred to $0 \mu \mathrm{V}^{2} / \mathrm{Hz}$. For the relaxed state, the minimum value of AsI is -0.24 and the maximum value of AsI is 0.48 while for the non-relaxed state the minimum value is -0.97 and the maximum value is 0.36 . Basically, the AsI values plotted between relaxed and non-relaxed states did not show an obvious difference.

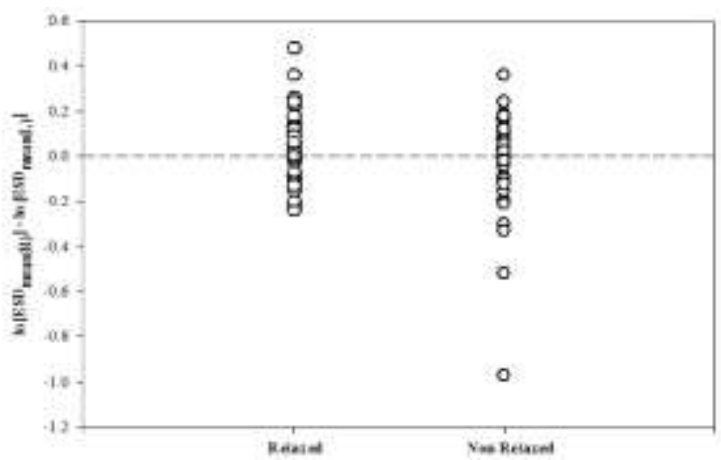

Fig. 4: Asymmetry index during relaxed state and non-relaxed state 


\subsection{Fuzzy C-Means Clustering}

The result from SC was used to estimate the initial cluster center in the iterative optimization-based FCM clustering algorithm. Thus, in the study there are five possible accessible cluster centers generated from the SC process. The resulting of the cluster center number was used as the preset number in the computation of FCM. However, in this study, the FCM preset cluster number was set to three. The selected data points to be in the same group based on the nearest distance from the cluster center and were marked with a similar symbol according to the groups. Each cluster's center which also known as the centroid is the average distance from the total data points within the same group.

As shown in Fig. 5 for the three clusters, different symbols have been generated that follow their respective cluster. From the results, the lesser the cluster centers, the farther the distances between the clusters centers and the lessen counts needed for the algorithm to converge. The aim was to have more than two different groups to establish the calmness indices. At the present, most of the studies only focusing on two different indices [11]. Thus, the three clusters analysis was selected in the study.

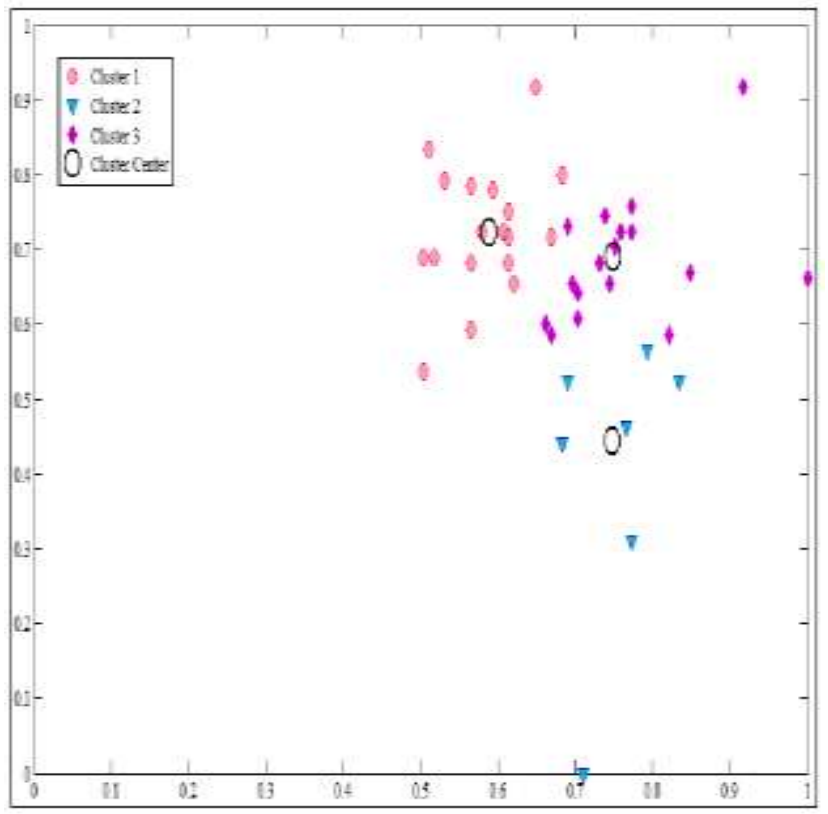

Fig. 5: Three clusters generated by FCM clustering

\subsection{EEG Pattern Verification}

Box plot was selected to confirm the validity of the clusters chosen by FCM. In this sub-section, the strength of EEG data was observed and discussed specifically of each cluster selected by FCM in three cluster groups as shown in Fig. 6. FCM clustered the approximate behavior, which consists of relaxed state and nonrelaxed state in each cluster. The data distribution for most of the clusters was not normal, as the median line did not fall at the center of the box. The data were also skewed as the whiskers at the box were not balance. However, it can be observed that relaxed state performs higher response than the non-relaxed state.

From the boxplot results, a nonparametric test should be recommended to be used in establishing the calmness indices. To facilitate the confirmation of the data distribution, a numerical statistical test need to be executed. Therefore, a comparable test with paired sample t-test for non-normal data is the Wilcoxon Signed Ranks test. Wilcoxon Signed Ranks test was chosen to further analyse a small sample of data. Wilcoxon Signed Ranks test used a hypothesis testing, which using the same method as paired sample t-test.

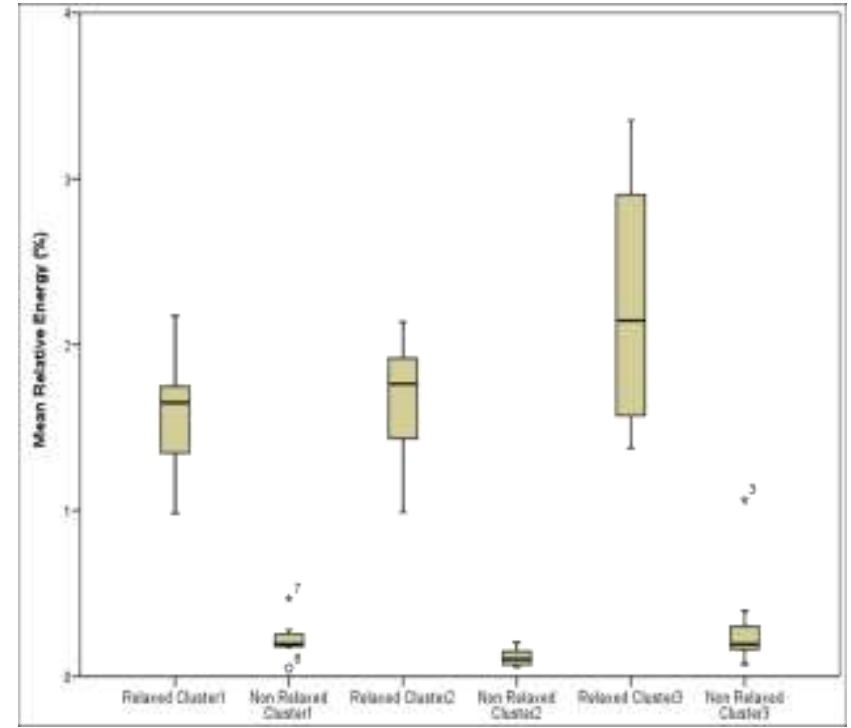

Fig. 6: Box plot patterns for three clusters

The results of Wilcoxon Signed Ranks test produce the rank summary and results of the significant value and the $\mathrm{Z}$-value. In the study, the $\mathrm{p}$ value was set to be 0.05 which is equivalent to $95 \%$ confidence level. Thus, the p-value to be achieved must be equivalent or less than 0.05 to prove that the data between the relaxed state and non-relaxed state is significant. While, the value of $\mathrm{Z}$, should be exceeded 1.96 or in this study was rounded to 2 in order to gain the robustness as paired sample t-test in the parametric test.

The overall results will only be accepted as a set of the calmness index if all clusters achieved significant results. This is because all significant clusters will be assigned as a meaningful index of calmness. Table 2 shows the results generated from the Wilcoxon Signed Rank test. All clusters have successfully accomplished significant results. This was evidenced by achieving $\mathrm{p}=0.012$ and $\mathrm{Z}=-2.524$ for Cluster 1 , followed by $\mathrm{p}=0$ and $\mathrm{Z}=-3.621$ for Cluster 2 and lastly for Cluster $3, p=0.002$ and $Z=-3.070$. The $p-$ values and the $\mathrm{Z}$-values obtained from the Wilcoxon Signed Ranks test for three clusters implied that there were significant differences between the relaxed state and non-state relaxed, which contained in each cluster. Thus, the three clusters were also feasible to be used as an index of calmness.

Table 2: Results of Wilcoxon signed rank test for three clusters

\begin{tabular}{|c|c|c|}
\hline & \multicolumn{2}{|c|}{ Value } \\
\hline Cluster 1 & -2.524 \\
\cline { 2 - 3 } (Relaxed - Non Relaxed) & Asymp. Sig. (2-tailed) & .012 \\
\hline Cluster 2 & $\mathrm{Z}$ & -3.621 \\
\cline { 2 - 3 } (Relaxed - Non Relaxed) & Asymp. Sig. (2-tailed) & .000 \\
\hline Cluster 3 & $\mathrm{Z}$ & -3.070 \\
\cline { 2 - 3 } (Relaxed - Non Relaxed) & Asymp. Sig. (2-tailed) & .002 \\
\hline
\end{tabular}

\subsection{EEG Calmness Indexing - $Z$ score}

In the Wilcoxon Signed Rank test, one of the generated results is the $\mathrm{Z}$ value or the $\mathrm{Z}$-score. The $\mathrm{Z}$-score values are used in the formation of the calmness indices from the lower index to higher index. As mentioned, using $\mathrm{Z}$-score will enable one to determine the accurate positions of the distribution means within the data in the cluster. Fig. 7 showed the Z-score trends for three clusters. The efficiency of the non-parametric test was set to $Z>1.96$ or simplified to 2.0 , which indicate significant discrimination between the relaxed and non-relaxed states. From the figure, the value of Zscores proved diversity of indices. The lowest calmness index is measured from the line, which is equivalent to $2 \sigma$. The lowest degree of Z-score is Cluster 1, followed by Cluster 3 and the highest degree is obtained at Cluster 2. 


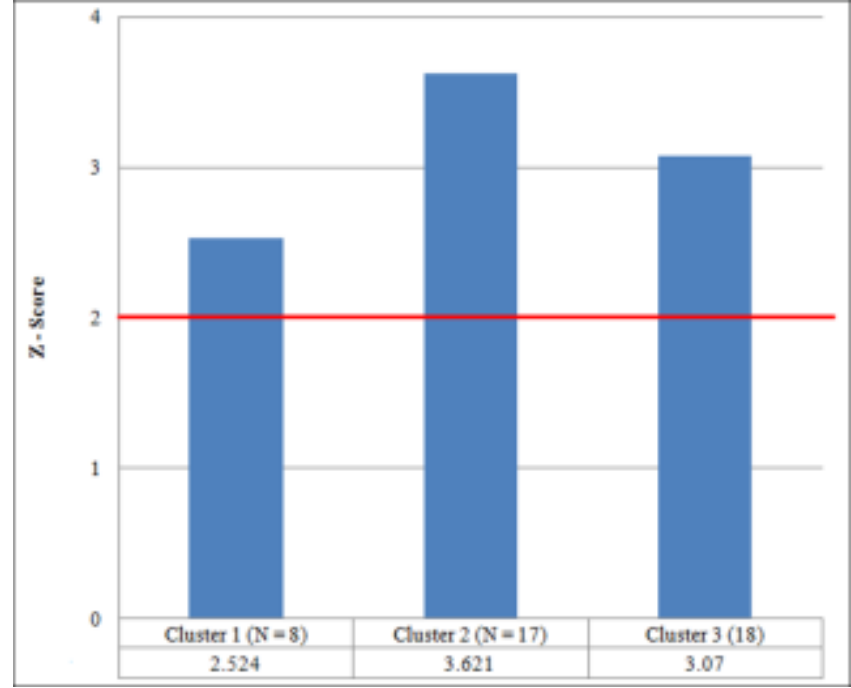

Fig. 6: Plot of Z-score for three calmness indices

The results are evaluated from the lowest significant difference to the highest significant difference in the three clusters has been simplified in Table 3. The $Z$-scores values of $Z_{1}=2.524, Z_{3}=3.07$ and $Z_{2}=3.621$ which are the $Z$-scores at Cluster 1 , Cluster 3 and Cluster 2 respectively. The highest relax index occurred above $3 \sigma$ which is approximate $99.87 \%$.

Table 3: Three degree of calmness indices

\begin{tabular}{|c|c|c|c|}
\hline \multicolumn{2}{|c|}{ Z-Score Indicator } & Cluster & Level of Calmness \\
\hline Lowest & 2.534 & 1 & 1 \\
\hline Moderate & 3.07 & 3 & 2 \\
\hline Highest & 3.621 & 2 & 3 \\
\hline
\end{tabular}

\section{Conclusion}

In this study, the collected EEG data were pre-processed and computed before it was used as the EEG features. The preprocessed EEG signals were confirmed its quality in order to ensure the collected EEG signals were highly discriminated between two conditions namely EEG relaxed state and EEG non-relaxed state. The statistical analysis was used to validate the EEG trend and to confirm it was distinctly separated. The results obtained have indicated that the relaxed state and the non-relaxed state were statistically significant.

The Z-score produced by Wilcoxon Signed Rank test has been applied in establishing the calmness index. The minimum value was set to $Z=2.0$ to be accepted as the calmness index. This is because, during $Z=2.0$, the $\mathrm{p}$-value $<0.05$ which was significant and comparable with the parametric test. Hence, for the study, the $\mathrm{Z}$-scores in the three clusters have fulfilled the evaluation and can be proposed as the calmness indices. The study has proven that the mental conditions of an individual can be categorized and indexed if appropriate methods were used.

\section{Acknowledgement}

The authors would like to thank the Razak Faculty of Technology and Informatics, Universiti Teknologi Malaysia for the sponsorship of the study under research grant no.: R.K130000.7340.4J301 and also to all collaborating partners.

\section{References}

[1] 1. Subha, D.P., P.K. Joseph, R. Acharya, and C. M, Lim, EEG signal analysis: A survey. Journal of Medical Systems, 2010, 34(2): 195-212.

[2] 2. Jackson, C.E. and P.J. Snyder, Electroencephalography and event-related potentials as biomarkers of mild cognitive impairment and mild Alzheimer's disease. Alzheimer's and Dementia, 2008, 4(1, Supplement 1): S137-S143.

[3] 3. Finnigan, S.P., S.E. Rose, M. Walsh, M. Griffin, A.L. Janke, K.L. McMahon, R. Gillies, M.W. Strudwick, C.M. Pettigrew, J. Semple, and J. Brown, Correlation of quantitative EEG in acute ischemic stroke with 30-day NIHSS score: Comparison with diffusion and perfusion MRI. Stroke, 2004, 35(4): 899-903.

[4] 4. Klimesch, W., EEG alpha and theta oscillations reflect cognitive and memory performance: A review and analysis. Brain Research Reviews, 1999, 29(2-3): 169-195.

[5] 5. Lal, S.K.L. and A. Craig, A critical review of the psychophysiology of driver fatigue. Biological Psychology, 2001, 55(3): 173-194.

[6] 6. La Vaque, T.J., The history of EEG Hans Berger: Psychophysiologist. A historical Vignette. Journal of Neurotherapy, 1999, 3(2): 1-9.

[7] 7. Barry, R.J., A.R. Clarke, R. McCarthy, M. Selikowitz, J.A. Rushby, and E. Ploskova, EEG differences in children as a function of resting-state arousal level. Clinical Neurophysiology, 2004, 115(2): 402-408.

[8] 8. Sigl, J.C. and N.G. Chamoun, An introduction to bispectral analysis for the electroencephalogram. Journal of Clinical Monitoring, 1994, 10(6): 392-404.

[9] 9. Kusserow, M., O. Amft, and G. Troster, Monitoring stress arousal in the wild. Pervasive Computing, 2013, 12(2): 28-37.

[10] 10. Hinrikus, H., A. Suhhova, M. Bachmann, K. Aadamsoo, Ü. Võhma, J. Lass, and V. Tuulik, Electroencephalographic spectral asymmetry index for detection of depression. Medical and Biological Engineering and Computing, 2009, 47(12): 1291-1299.

[11] 11. Musha, T., S. Kimura, K.I. Kaneko, K. Nishida, and K. Sekine, Emotion spectrum analysis method (ESAM) for monitoring the effects of art therapy applied on demented patients. CyberPsychology and Behavior, 2000, 3(3): 441-446.

[12] 12. Thatcher, R.W., Latest developments in live Z-score training: Symptom check list, phase reset, and Loreta Z-score biofeedback. Journal of Neurotherapy, 2013, 17(1): 69-87.

[13] 13. Lias, S., Z. Murat, N. Sulaiman, and M. N. Taib, IQ index using alpha-beta correlation of EEG power spectrum density (PSD). Proceedings of the IEEE Symposium on Industrial Electronics and Applications, 2010, pp. 612-616.

[14] 14. Ismail, N., M. H. F.1 Rahiman, M. N. Taib, N. A. M. Ali, M. Jamil, and S. N. Tajuddin, Classification of the quality of agarwood oils from Malaysia using Z-score technique. Proceedings of the International Conference on System Engineering and Technology, 2013, pp. 78-82.

[15] 15. Jain, A., K. Nandakumar, and A. Ross, Score normalization in multimodal biometric systems. Pattern Recognition, 2005, 38(12): 2270-2285.

[16] 16. Cheadle, C., M.P. Vawter, W.J. Freed, and K.G. Becker, Analysis of microarray data using $\mathrm{Z}$ score transformation. Journal of Molecular Diagnostics, 2003, 5(2): 73-81.

[17] 17. Ramirez, R.W., The FFT fundamentals and concepts. 1985, Prentice-Hall.

[18] 18. Bellera, C.A., M. Julien, and J.A. Hanley, Normal approximations to the distributions of the Wilcoxon Statistics: Accurate to what N? Graphical Insights. Journal of Statistics Education, 2010, 18(2): 1-17.

[19] 19. Sullivan, M., Statistics: Informed decisions using data. 2004, Pearson Education. 